\title{
Current Status of Soil-Transmitted Helminths among School Children in Kakamega County, Western Kenya
}

\author{
Teresia Ngonjo, ${ }^{1}$ Collins Okoyo, ${ }^{2}$ Julius Andove, ${ }^{2}$ Elses Simiyu, ${ }^{2}$ Agola Eric Lelo, ${ }^{2,3}$ \\ Ephantus Kabiru, ${ }^{4}$ Jimmy Kihara, ${ }^{2,5}$ and Charles Mwandawiro ${ }^{2}$ \\ ${ }^{1}$ Karatina University, P.O. Box 1957-10101, Karatina, Kenya \\ ${ }^{2}$ Kenya Medical Research Institute (KEMRI), Eastern and Southern Africa Centre of International Parasite Control, \\ P.O. Box 54840-00200, Nairobi, Kenya \\ ${ }^{3}$ Technical University of Kenya (TUK), P.O. Box 52428-00200, Nairobi, Kenya \\ ${ }^{4}$ Kenyatta University, P.O. Box 43844-00202, Nairobi, Kenya \\ ${ }^{5}$ Division of Vector Borne Diseases, Ministry of Health, P.O. Box 20750-00202, Nairobi, Kenya
}

Correspondence should be addressed to Teresia Ngonjo; ngonjo_teresia@yahoo.com

Received 17 February 2016; Revised 20 May 2016; Accepted 24 May 2016

Academic Editor: Emmanuel Serrano Ferron

Copyright (c) 2016 Teresia Ngonjo et al. This is an open access article distributed under the Creative Commons Attribution License, which permits unrestricted use, distribution, and reproduction in any medium, provided the original work is properly cited.

Background. School age children are at high risk of soil-transmitted helminth (STH) worldwide. In Kenya, STH infections in children remain high despite the periodic administration of anthelmintic drugs. Our study assessed the prevalence and intensity of STH in primary school-aged children in Kakamega County, western Kenya. Methodology. We carried out a cross-sectional study on a population of 731 children attending 7 primary schools in March 2014. Children aged 4-16 years were examined for STH by the quantitative Kato-Katz technique. Infection intensities were expressed as eggs per gram (epg) of faeces. Findings. Among 731 school children examined for STH, 44.05\% were infected. Highest prevalence of STH was in Shitaho primary school where 107 participants were examined and $62.6 \%$ were infected with mean intensity of $11667 \mathrm{epg}$. Iyenga had the least prevalence where 101 participants were examined and $26.7 \%$ were infected with mean intensity of $11772 \mathrm{epg}$. A. lumbricoides was the most prevalent STH species with $43.5 \%$ infected, while hookworm infections were low with $1.8 \%$ infected. Conclusion. Prevalence of STHs infections in Kakamega County remains high. We recommend guidelines and other control strategies to be scaled up to break transmission cycles.

\section{Introduction}

Soil-transmitted helminths (STHs) infections represent a major public health problem in poor and developing countries and have constituted a universal burden which does depend not only on regional ecological condition but also on local standard of social and economic development of the people [1]. Many different species of soil-transmitted helminths infect humans, especially in the tropical and subtropical parts of the developing world [2]. Four nematodes, in hundreds of millions of human infections, in particular stand out because of their widespread prevalence. These include roundworm, Ascaris lumbricoides, the whipworm Trichuris trichiura, and two species of hookworm, Necator americanus and Ancylostoma duodenale [2].
Today, it is estimated that approximately one-third of the almost three billion people that live on less than two US dollars per day in developing regions of Sub-Saharan Africa, Asia, and the Americas are infected with one or more helminths $[3,4]$. The most common helminthiases are those caused by intestinal helminths, ascariasis, trichuriasis, and hookworm. This means that the inhabitants of thousands of rural, impoverished villages throughout the tropics and subtropics are often chronically infected with several different species of parasitic worm; that is, they are polyparasitized $[3,4]$.

For reasons not well understood, compared with any other age group, school-aged children (including adolescents) and preschool children tend to harbor the greatest numbers of intestinal worms and as a result experience growth stunting and diminished physical fitness as well as 
impaired memory and cognition [5]. These adverse health consequences combine to impair childhood educational performance, reduce school attendance [6], and account for the observation that hookworm and presumably other diseases caused by parasitic worms reduce future wage-earning capacity [7].

Global commitment focuses on school based chemotherapy programmes to implement helminth control strategies [8]. Currently it is estimated that more than 2 billion people worldwide are infected with STH. As discussed elsewhere, the majority of this population resides in low and middle income countries (LMICs) $[7,8]$. Together with LMICs, STH endemicity is concentrated in areas where ecological and environmental characteristics facilitate transmission $[8,9]$.

Global numbers infected with STH infection will remain an elusive goal, due in part to a scarcity of reliable and accurate epidemiological data and in part lack of specificity of clinical signs due to STH $[10,11]$. Ministry of Health's Department of Child Health in Kenya seeks to promote good health and nutrition. The ministry recognizes the detrimental effects of STH infections in primary-school-aged children [12]. School children serve in assessment of helminths distribution and prevalence. School based deworming programmes provide information and help to design implementation of cost-effective control programme that aims to monitor STH impact at both national and regional levels. The objective of this cross-sectional study was to determine the prevalence of soil-transmitted helminths infections among school children in informal urban settlements in Kakamega, Western Kenya, prior to a National Deworming Mass Drug Administration.

\section{Materials and Methods}

2.1. Study Site. The study was carried out in Kakamega County. It is located at $00^{\circ} 20^{\prime} \mathrm{N} 34^{\circ} 46^{\prime} \mathrm{E}$ with an area of $3,033.8 \mathrm{~km}$. The climate is mainly tropical, with variations due to altitude. Kakamega County is mainly cool and wet most of the year. The county has a mixture of both subsistence and cash crop farming, with sugar cane being the preferred largescale cash crop. It has a population of $1,660,651$ people [13].

2.2. Study Population. Pupils in early childhood, classes two to six from Kakamega East, South, and Central Sub-Counties, formed the study group. Pupils from schools that have not been dewormed in the last year and those who have been dewormed six months in the National Deworming Programme prior to the commencement of the study were included. Those who have been dewormed within the three months were not included. Children below 4 years and above 16 years were not included in the study. A list of registered pupils in each class was obtained from the head teacher in each school and age of each participating pupil was confirmed from parent when obtaining the informed consent. Parents and guardians consented to have the results of the study published while adhering to the privacy of the study subjects.

2.3. Study Design and Sampling. Seven schools were randomly selected in Kakamega County. A total of 731 children in 7 primary schools in 3 sub-counties of Kakamega County were recruited in the study. At each selected school, 9 boys and 9 girls were randomly selected from classes 2 to 6 and early childhood to have a total of 108 children per school. This was a cross-sectional study to assess the prevalence and intensity among the sampled children before mass drug administration (MDA) in school-aged children in the study area.

2.4. Parasitological Examination. Each participating child was asked to provide fresh stool sample for Kato-Katz examination to detect soil-transmitted helminths infections. Each pupil was guided on how to provide about 2 grams of stool samples. Diagnosis of helminths was determined by presence of egg counts of soil-transmitted helminths (STHs) in defined quantities of stool using Kato-Katz technique [14]. Two Kato slides per stool sample were prepared using fixed quantity of sieved $41.7 \mathrm{mg}$ (WHO kit) of stool on a punched template. It was then mounted on slides and covered with malachite green impregnated cellophane. The slides were observed within one hour under the microscope at a magnification of $\times 10$. For hookworms, the slides were read immediately while Ascaris lumbricoides and Trichuris trichiura eggs were examined 60 minutes later. The total numbers of eggs were expressed as eggs per gram (epg) of faeces. Quality control was performed by systematic random examination, by the team leader of $10 \%$ of the daily examined Kato-Katz slides.

2.5. Statistical Analysis. Observed prevalence of each STH species was calculated at school, sub-county, and regional levels, and 95\% confidence intervals (95\% CIs) were obtained by binomial logistic regression, taking into account clustering by schools. Comparisons of prevalence by location, age group, and sex were tested for significance on the basis of the Wald test. For the purposes of analysis, the following age groups were used: $3-5,6-7,8-9,10-11,12-13$, and above 13 years old. Mean egg counts were expressed as arithmetic mean epg and since egg counts are not normally distributed, 95\% CIs were estimated using a negative binomial regression model. Infection intensities were classified into light to heavy intensity of infections according to WHO guidelines [15], and the prevalence of light to heavy infections and 95\% CIs adjusted for school clusters were also obtained using binomial regression.

\section{Results}

Overall, data was collected from 731 children in 7 primary schools in 3 sub-counties of Kakamega County. Of 731 participants, $28.5 \%$ of the pupils were from Kakamega South Sub-County, 43.1.\% from Kakamega East Sub-County, and 28.9\% from Kakamega Central Sub-County. The children had almost equal representation in terms of gender. Of the 731 participants examined, $50.2 \%$ were males and $49.8 \%$ females. The mean age of pupils was 10 years $(\mathrm{SD}=2.6$ years $)$ with age range of 4 years to 16 years (Figure 1).

3.1. Overall Prevalence and Intensity. Of the 731 participants examined for STHs combined, 44.05\% (95\% CI: 35.80-54.20) were infected. A. lumbricoides was the most prevalent STH 


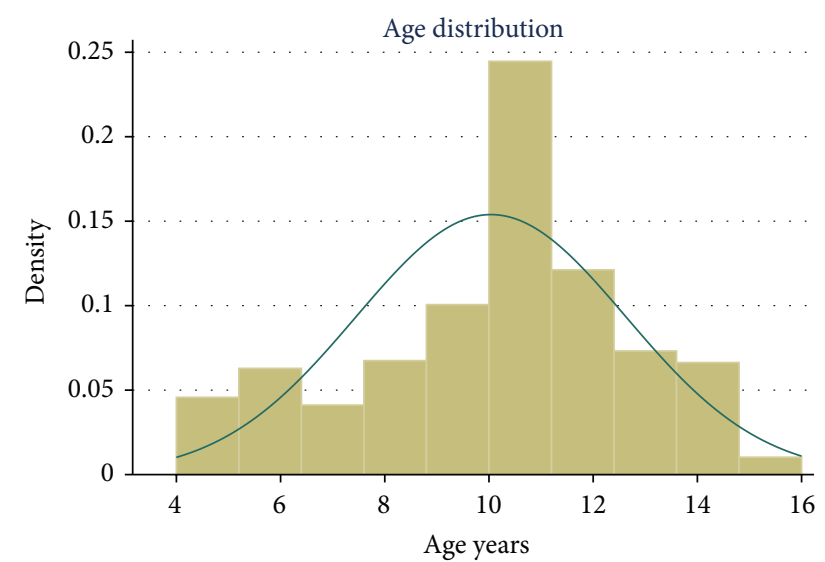

FIGURE 1: The age distribution of the children sampled for the study.

TABLE 1: Overall prevalence and mean intensity of STH infections.

\begin{tabular}{lcc}
\hline Infection & Prevalence $(\%)$ & $95 \%$ CI \\
\hline STHs combined & 44.05 & $(35.80-54.20)$ \\
Hookworm & 0.27 & $(0.06-1.09)$ \\
A. lumbricoides & 43.5 & $(35.21-53.74)$ \\
T. trichiura & 0.82 & $(0.38-1.78)$ \\
\hline Infection & Mean intensity (epg) & $95 \%$ CI \\
\hline STHs combined & 3674 & $(2560-5274)$ \\
Hookworm & 0.33 & $(0.05-2.29)$ \\
A. lumbricoides & 3673 & $(2559-5274)$ \\
T. trichiura & 0.84 & $(0.16-4.38)$ \\
\hline
\end{tabular}

species. Out of the total participants, $43.5 \%$, (95\% CI: $35.21-$ 53.74) were infected. Hookworm and T. trichiura had generally low prevalence. Of those examined, $0.27 \%$, (95\% CI: $0.06-1.09)$ and $0.82 \%$, (95\% CI: $0.38-1.78)$ were infected, respectively (Table 1 ).

The overall mean intensity of the combined STH species was $36743 \mathrm{epg}$, (95\% CI: 2560-5274). A. lumbricoides had the highest mean intensity of $3673 \mathrm{epg}$, (95\% CI: 2559-5274); hookworm and T. trichiura had generally low mean intensity of $0.33 \mathrm{epg}$, (95\% CI: 0.05-2.29) and $0.84 \mathrm{epg}$, (95\% CI: 0.164.38 ), respectively (Table 1 ).

\subsection{Soil-Transmitted Helminths (STHs) Distribution within} Schools and Sub-County. The prevalence and mean intensity for any STHs species were examined for surveyed schools, sub-county, gender, class, and age groups taking into account clustering by schools.

Overall, among the 7 schools surveyed, Shitaho primary school had the highest prevalence of STHs combined. Of the 107 participants examined, 62.6\% (95\% CI: 54.1-72.5) were infected with mean intensity 11667 epg (95\% CI: 8400-16205). Bukusi primary school had 99 participants; $55.6 \%$, (95\% CI: 46.6-66.3) were infected with mean intensity 7368 epg (95\% CI: 4827-11246). Iyenga primary had the least prevalence. Of the 101 participants examined, $26.7 \%$ (95\% CI: 19.4-36.9) were infected with mean intensity of 11772 epg (95\% CI: 688920115) (Table 2).
Hookworm was only observed in Bukhulanya primary school. Of the 110 participants in that school, 1.8\% (95\% CI: $0.5-7.2)$ were infected with mean intensity of 120 epg (95\% CI: 41-355). Kakamega Central had the highest prevalence of STHs combined. Of the 211 participants examined, 52.1\% (95\% CI: 34.9-77.8) were infected with mean intensity of 10768 epg (95\% CI: 8822-13144). In the same sub-county, 52.1\% (95\% CI: 35-77.8) were infected with Ascaris lumbricoides with mean intensity of $10763 \mathrm{epg}$ (95\% CI: 8808-13153). Only $0.9 \%$, (95\% CI: 0.1-6.9) of the participants examined in Kakamega Central were infected with T. trichiura with mean intensity 264 epg (95\% CI: 39-1804). However, hookworm was only prevalent in Kakamega South district. Of the 208 participants examined, $0.9 \%$ (95\% CI: (0.1-6.2) were infected with hookworm with mean intensity of 120 epg (95\% CI: 41355) (Table 3).

3.3. Soil-Transmitted Helminths (STHs) Distribution within Gender and Age Categories. The prevalence of STH combined infection and any single STHs did not vary markedly between males and females participants (Table 4). Generally, children with 4-5 years showed highest prevalence. Of the total examined, 67.5\% (95\% CI: 55.5-82.1) were infected for STH combined and A. lumbricoides species compared to other age groups. However, low levels of hookworm prevalence were present among (6-7) years and (12-13) years (Figure 2).

Children with age (4-5) years had the highest prevalence of STHs combined (67.5\%) compared to other age groups, whereas the mean intensity of STHs combined was high in the age groups (6-7) years and (12-13) years, 10,752 epg and 9,217 epg, respectively (Figure 3 ).

Similarly, prevalence of $A$. lumbricoides was highest among pupils of (4-5) years, $67.5 \%$ with other age categories showing prevalence ranging between $45 \%$ and $40 \%$. Generally, there were high levels of A. lumbricoides mean intensity in age groups (6-7) and (12-13) years. Children above 13 years had the least mean intensity of A. lumbricoides, 4,241 epg (Figure 3). Low levels of T. trichiura prevalence were only found in age groups (8-9), (12-13), and (>13) while the remaining age groups had no T. trichiura infection. Low levels of mean intensity of infection were only recorded in age groups (8-9) and (>13) years (Figure 3 ).

3.4. Light-Heavy Intensity of Intestinal Helminths. Intestinal infections were categorized from light-heavy intensities according to WHO guidelines [15]; however, only A. lumbricoides intensity could fit into more than one category, while the remaining infections studied had light intensities. 80.6\% of the pupils had light intensity of A. lumbricoides while $19.4 \%$ had moderate intensity.

\section{Discussion}

The study assessed the prevalence of three soil-transmitted helminths infections in school children in three sub-counties, namely, Kakamega Central, Kakamega South, and Kakamega East. The overall STHs infections had varied prevalence in the different schools and sub-counties. It was evident that 


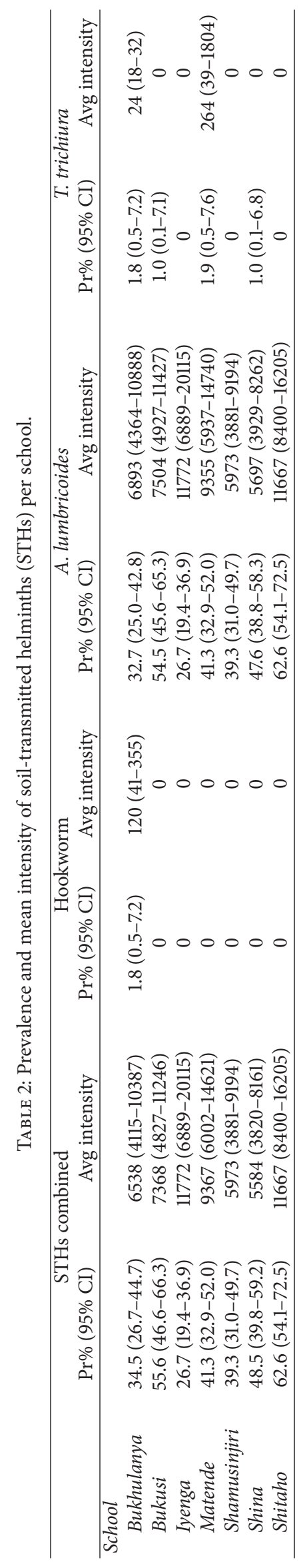




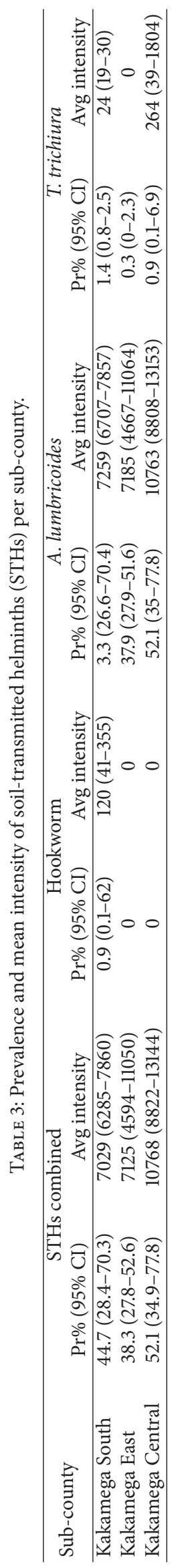


TABLE 4: Prevalence and mean intensity of soil-transmitted helminths (STHs) per gender.

\begin{tabular}{lccccccccc}
\hline & \multicolumn{2}{c}{ STHs combined } & \multicolumn{2}{c}{ Hookworm } & \multicolumn{2}{c}{ A. lumbricoides } & \multicolumn{2}{c}{ T. trichiura } \\
Gender & $\operatorname{Pr} \%(95 \% \mathrm{CI})$ & Avg intensity & Pr\% (95\% CI) & Avg intensity & Pr\% (95\% CI) & Avg intensity & Pr\% (95\% CI) & $\begin{array}{c}\text { Avg } \\
\text { intensity }\end{array}$ \\
\hline Male & $44.1(37.2-52.4)$ & $7150(5474-9339)$ & $0.3(0-1.8)$ & 0 & $43.3(36.4-51.5)$ & $7281(5647-9386)$ & $1.4(0.6-2.9)$ & $(32-432)$ \\
Female & $44.0(32.6-59.3)$ & $9549(7250-12577)$ & $0.3(0-2.0)$ & 0 & $43.7(32.2-59.3)$ & $9607(7312-12624)$ & $0.3(0-2.0)$ & 0 \\
\hline
\end{tabular}
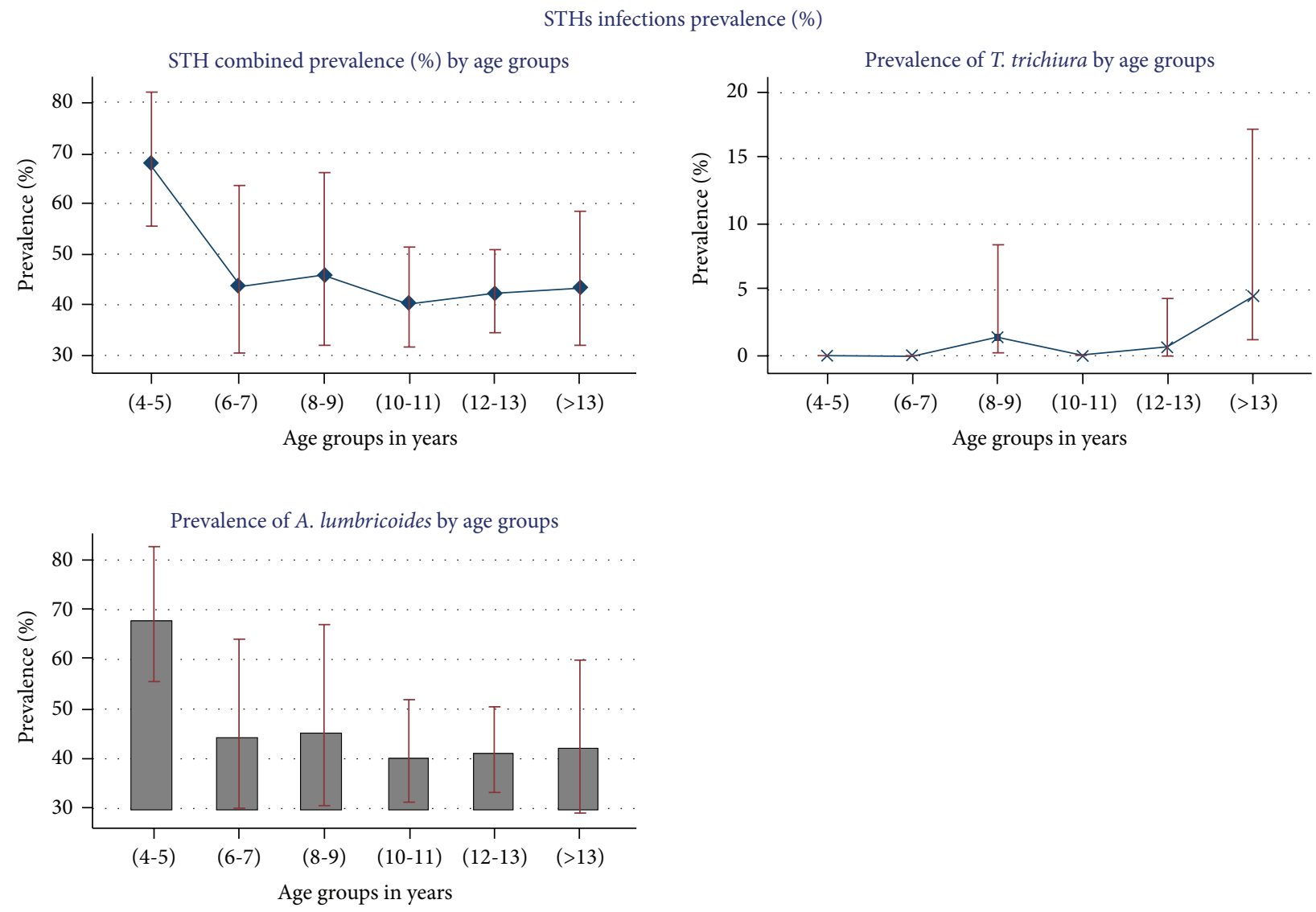

FIGURE 2: Prevalence (\%) of various STHs species by age category.

Kakamega Central Sub-County had the highest prevalence (52.1\%) of STHs infections. This was far much higher than what was reported in the neighbouring Sub-County Kisumu East, in Nyanza County (17.4\%), and Kilindini in Mombasa County (18.0\%) [16]. The most common STHs detected among children were $A$. lumbricoides, followed by very low prevalence $(0.9 \%)$ of $T$. trichiura. However, hookworm was only reported in Kakamega South Sub-County with low levels of prevalence reported among children of ages (6-7) years and (12-13) years. This suggests low exposure to infective hookworm larvae that commonly occurs outside the household like in agricultural areas or in defined defecation sites [17]. There was low T. trichiura prevalence in Bukhulanya, Matende, Bukusi, and Shina primary schools. This showed that there is a remarkable decrement in the prevalence in some of parasitic infections. The progress in the reduction of the parasites prevalence could be due to different interventions that have been undertaken in the area like national school based deworming and health education. Differences between sites may be explained by the relative importance of domains in defining contact with infectious stages [18].

The prevalence of STHs combined and any single STHs did not vary markedly between males and females participants. These results agree with those from a study conducted in the central part of Turkey that showed there was no statistically significant difference observed between presence of intestinal prevalence and gender [19]. Overall, high prevalence and intensities were observed in age group 4-5 years $(67.5 \%)$ compared to other age groups, whereas the mean intensity of STHs combined was high in the age groups (6-7) years and (12-13) years, 10,752 epg and 9,217 epg, respectively. These results were different from those of Kibera where $16.2 \%$ 
STHs infections mean intensity (epg)
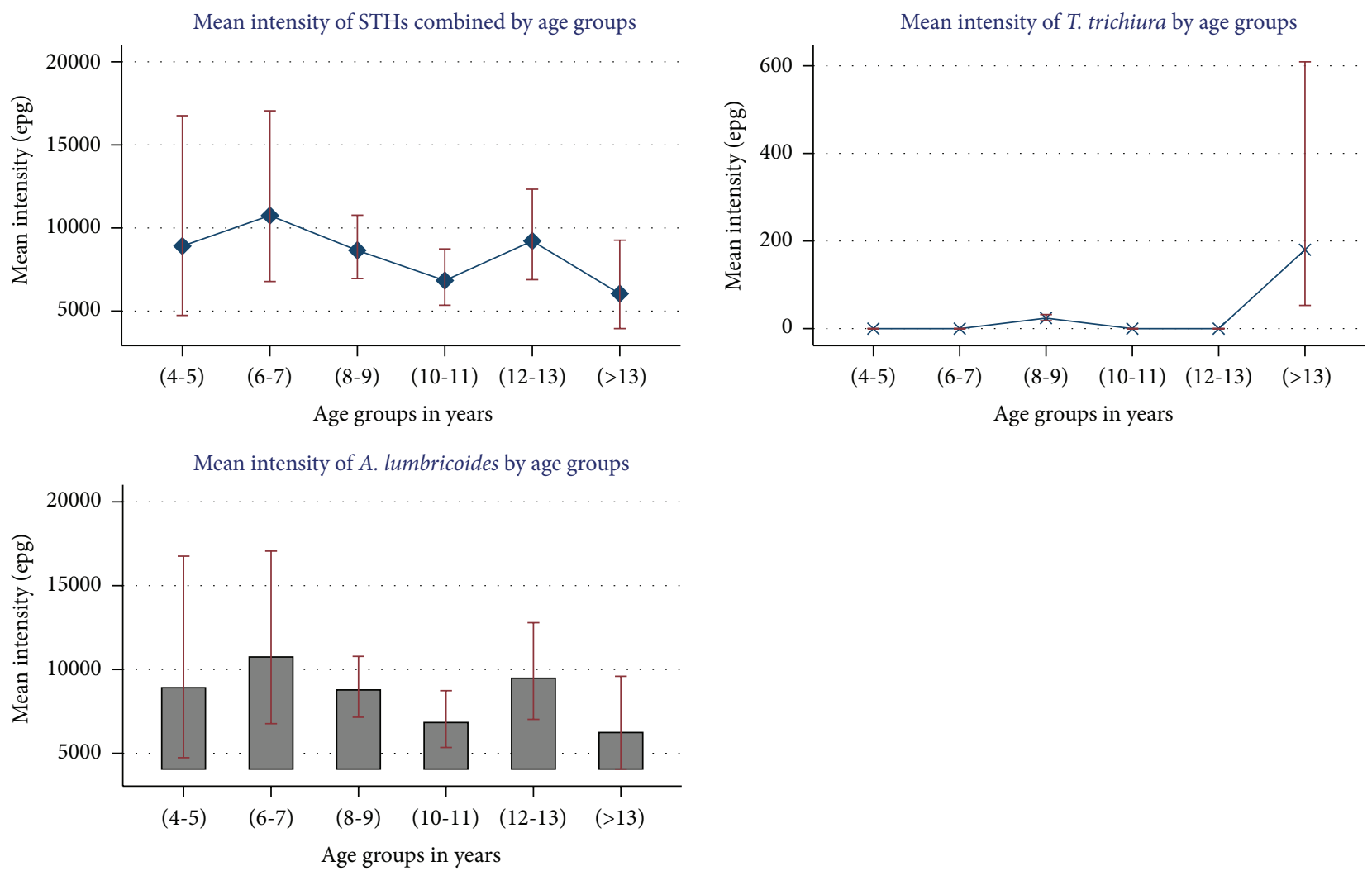

FIgURE 3: Mean intensity (epg) of various STHs species by age category.

prevalence was reported among children aged 10-18 years living in urban slum settings in Nairobi Kenya [20].

Prevalence of $A$. lumbricoides was high among pupils of (4-5) years which was $67.5 \%$ with other age categories showing prevalence ranging between $45 \%$ and $40 \%$. This prevalence was much higher than A. lumbricoides in Thika Sub-County, Kiambu County [21]. There were higher levels of A. lumbricoides mean intensity in age groups (6-7) and (12-13) years. Children above 13 years had the least mean intensity of A. lumbricoides. These results compare with a study carried out in Honduras where children aged 5 to 12 years had a higher percentage of $A$. lumbricoides infections than individuals in the other age groups. However, both the prevalence and intensity of $A$. lumbricoides varied significantly by age $(p<0.001)$.

The different prevalence of STHs in the study is in agreement with previous studies from Western Kenya, although higher percentage prevalence was noted for A. lumbricoides infections (43.5\%) in this study. Current study had low prevalence hookworm and T. trichiura, $0.27 \%$ and 0.82 , respectively. Informal settlements of Kisumu had prevalence of $4.9 \%$ for $A$. lumbricoides and $7.7 \%$ for $T$. trichiura [22]. In line with a recent STH survey in these regions, A. lumbricoides infections were more prevalent compared to $T$. trichiura and hookworm [23].

It was remarkable feature that this study showed that high numbers of children were infected with A. lumbricoides. It could be due to the fact that transmission of $A$. lumbricoides is through faecal oral route and infections after treatment reappear fast, particularly for A. lumbricoides [23]. Coinfections of STHs were not common in the study area. Multiple STH infections were less common than in other studies elsewhere in East Africa [16, 24]. In Busia, Kenya, 26\% of children were infected with all 3 STHs and $31.1 \%$ with 2 STHs [16]. Current study also differs with what was observed in Pemba, Tanzania, where $67 \%$ of children were infected with all 3 STHs and $28 \%$ with 2 STHs [25]. Such differences might have arisen due to differences in the study subjects, sociodemographic conditions, and socioeconomic characteristics of the areas. Coinfection may affect nutritional status of the children because of the combined effect of the different parasites that can deprive the children of the important nutrients. Again children with a single infection stand out with a better academic score than those with multiple infections [26].

Our findings confirm that the children did not harbor multiple infections of STHs. This is an important observation and could be attributed to impact of the school based approach adopted control programme. Low prevalence and mean intensity of hookworm and T. trichiura in this study are an implication that mass treatment has reduced their refugia and the rate of infection was low. However, the prevalence of A. lumbricoides is significantly high. This is an endemic area where there is high daily occurrence of transmission because of soil and food contaminated with infective stages 
of this parasite [27]. This could lead to high morbidity in the children and predisposing factors for this parasite require to be improved.

The mean intensities in hookworm and T. trichiura were quite low and were all in light infection. This indicates less contamination of the environment with infective larvae. Hookworm has a slower rate of infection compared to $A$. lumbricoides because its third-stage larvae have shorter life expectancy (3-10 days) unlike A. lumbricoides eggs with several months' infective period [28]. This implies that the environment can recover more quickly from hookworm contamination than from $A$. lumbricoides. This could have been attributed to the high prevalence of $A$. lumbricoides and its moderate-light infection in the current study. Reduced prevalence of hookworm and $T$. trichiura could also be attributed to high community level access to improved sanitation, as well as county economy and health service delivery indicator scores. School based intervention should be established in order to reduce infection rates.

When comparing our baseline survey results to historical data, STH infection prevalence has strongly decreased over the last decade in certain regions of Kenya where a deworming programme has been implemented since 1998 $[29,30]$. However, Kakamega County in Western Kenya has remained a high endemic area. Our study did not assess risk factors associated with STH. However, it is evident from our results that prevalences of STH were high in this area, an indication that transmission is high and could be influenced by conditions that determine the development and survival of free-living stages in the external environment [28]. Survival of STH free-living stages is dependent on environmental conditions that influence their transmission success. Limited access to water, sanitation, and hygienic behaviour determines the rate of exposure to ova and larvae [31].

\section{Conclusion}

The analysis of collected data provided useful insight into the current prevalence and mean intensity of soil-transmitted helminths (STHs) infections in Kakamega County. A. lumbricoides is the predominant STH in the region. We advocate for integrated, multisectoral approaches prioritizing communities where focal transmission shows singular patterns. Factors that facilitate STH transmission in this region and their impact on prevalence and intensity should be considered in future surveys.

\section{Ethical Approval}

The study protocol received ethical approval from Kenyatta University Ethical Review Committee (PKU/150/1 131). Additional approval was provided by the appropriate county-level health and education authorities, who were briefed about the survey.

\section{Consent}

At the school level, parental consent was based on passive, opt-out consent rather than written opt-in consent owing to the low risk and routine nature of the study procedures. Individual assent was obtained from each child before participation in the survey. Information was given to publish results without revealing identification of the participants.

\section{Competing Interests}

The authors declare that they have no competing interests.

\section{Authors' Contributions}

Teresia Ngonjo participated in the survey design, data collection, and development of the paper. Collins Okoyo participated in the data analysis. Julius Andove and Elses Simiyu were responsible for fieldwork supervision and contributed to the final paper. Jimmy Kihara, Agola Eric Lelo, Ephantus Kabiru, and Charles Mwandawiro were responsible for the study design, interpretation, and scientific guidance. All authors read and approved the final paper.

\section{Acknowledgments}

The authors are grateful to the Kenya School Based Deworming Programme and the County Ministries of Health and Education for their support for this work and to the pupils who participated and their parents who consented.

\section{References}

[1] O. M. Ukpai and C. O. Ugwu, "The prevalence of gastro-intestinal tract parasites in primary school children in Ikwuano Local Government Area of Abia State, Nigeria," Nigerian Journal of Parasitology, vol. 240, pp. 129-136, 2003.

[2] P. J. Hotez, D. H. Molyneux, A. Fenwick, E. Ottesen, S. E. Sachs, and J. D. Sachs, "Incorporating a rapid impact package for neglected tropical diseases with programs for HIV/AIDS, tuberculosis, and malaria," PLoS Medicine, vol. 3, article e102, 2006.

[3] P. J. Hotez, D. H. Molyneux, A. Fenwick et al., "Control of neglected tropical diseases," The New England Journal of Medicine, vol. 357, no. 10, pp. 1018-1027, 2007.

[4] D. W. T. Crompton and M. C. Nesheim, "Nutritional impact of intestinal helminthiasis during the human life cycle," Annual Review of Nutrition, vol. 22, pp. 35-59, 2002.

[5] E. Miguel and M. Kremer, "Worms: identifying impacts on education and health in the presence of treatment externalities," Econometrica, vol. 72, no. 1, pp. 159-217, 2004.

[6] S. Baird, J. H. Hicks, M. Kremer, and E. A. Miguel, Worms at Work: Long-Run Impacts of Child Health Gains, Department of Economics at the University of California, 2011.

[7] World Health Organization, Soil-Transmitted Helminthiases: Eliminating Soil-Transmitted Helminthiases as a Public Health Problem in Children. Progress Report 2001-2010 and Strategic Plan 2011-2020, World Health Organization, Geneva, Switzerland, 2012.

[8] P. J. Hotez, A. Fenwick, L. Savioli, and D. H. Molyneux, "Rescuing the bottom billion through control of neglected tropical diseases," The Lancet, vol. 373, no. 9674, pp. 1570-1575, 2009. 
[9] J. Andrus, M. E. Bottazzi, J. Chow et al., "Ears of the armadillo: global health research and neglected diseases in Texas," PLoS Neglected Tropical Diseases, vol. 7, no. 6, Article ID e2021, 2013.

[10] A. Alemu, A. Atnafu, Z. Addis et al., "Soil transmitted helminths and Schistosoma mansoni infections among school children in zarima town, northwest Ethiopia," BMC Infectious Diseases, vol. 11, article 189, 2011.

[11] World Health Organisation, Deworming for Health and Development. Report of the Third Global Meeting of the Partners for Parasite Control, World Health Organization, Geneva, Switzerland, 2005.

[12] S. M. Njenga, C. S. Mwandawiro, E. Muniu, M. T. Mwanje, F. M. Haji, and M. J. Bockarie, "Adult population as potential reservoir of NTD infections in rural villages of Kwale district, Coastal Kenya: implications for preventive chemotherapy interventions policy," Parasites and Vectors, vol. 4, article 175, 2011.

[13] Kenya National Bureau of Statistics, The 2009 Kenya Population and Housing Census, KNBS, 2009.

[14] N. Katz, A. Chaves, and J. Pellegrino, "A simple device for quantitative stool thick-smear technique in Schistosomiasis mansoni," Revista do Instituto de Medicina Tropical de São Paulo, vol. 14, no. 6, pp. 397-400, 1972.

[15] Committee WHO, Prevention and Control of Schistosomiasis and Soil-Transmitted Helminthiasis, World Health Organization Technical Report Series, 2002.

[16] S. Brooker, E. A. Miguel, S. Moulin, A. I. Luoba, D. A. P. Bundy, and M. Kremer, "Epidemiology of single and multiple species of helminth infections among school children in Busia District, Kenya," East African Medical Journal, vol. 77, no. 3, pp. 157-161, 2000.

[17] G. A. Schad, T. A. Nawalinski, and V. Kochar, "Human ecology and the distribution and abundance of hookworm populations," in Human Ecology and Infectious Diseases, Academic Press, New York, NY, USA, 1983.

[18] S. Cairncross, U. Blumenthal, P. Kolsky, L. Moraes, and A. Tayeh, "The public and domestic domains in the transmission of disease," Tropical Medicine and International Health, vol. 1, no. 1, pp. 27-34, 1996.

[19] A. Topcu and K. Ugurlu, "Distribution of intestinal parasites in primary schools Nigde and its surrounding according to age, sex and socioeconomic status," ActaParasitologica Turcica, vol. 25, pp. 254-257, 2001.

[20] P. S. Suchdev, S. M. Davis, M. Bartoces et al., "Soil-transmitted helminth infection and nutritional status among urban slum children in Kenya," American Journal of Tropical Medicine and Hygiene, vol. 90, no. 2, pp. 299-305, 2014.

[21] T. W. Ngonjo, J. H. Kihara, M. Gicheru, P. Wanzala, S. Njenga, and C. S. Mwandawiro, "Prevalence and intensity of intestinal parasites in school age children in Thika District, Kenya," African Journal of Health Sciences, vol. 21, no. 3-4, pp. 153-160, 2012.

[22] M. R. Odiere, S. Opisa, G. Odhiambo et al., "Geographical distribution of schistosomiasis and soil-transmitted helminths among school children in informal settlements in Kisumu City, Western Kenya," Parasitology, vol. 138, no. 12, pp. 1569-1577, 2011.

[23] T.-W. Jia, S. Melville, J. Utzinger, C. H. King, and X.-N. Zhou, "Soil-transmitted helminth reinfection after drug treatment: a systematic review and meta-analysis," PLoS Neglected Tropical Diseases, vol. 6, no. 5, article e1621, 2012.

[24] C. S. Mwandawiro, B. Nikolay, J. H. Kihara et al., "Monitoring and evaluating the impact of national school-based deworming in Kenya: study design and baseline results," Parasites and Vectors, vol. 6, article 198, 2013.

[25] M. Albonico, H. M. Chwaya, A. Montresor et al., "Parasitic infections in Pemba Island school children," East African Medical Journal, vol. 74, no. 5, pp. 294-298, 1997.

[26] J. D. Kvalsvig, R. M. Cooppan, and K. J. Connolly, “The effects of parasite infections on cognitive processes in children," Annals of Tropical Medicine and Parasitology, vol. 85, no. 5, pp. 551-568, 1991.

[27] L. M. Vandemark, T.-W. Jia, and X.-N. Zhou, "Social science implications for control of helminth infections in Southeast Asia," Advances in Parasitology, vol. 73, pp. 137-170, 2010.

[28] S. Brooker, A. C. A. Clements, and D. A. P. Bundy, "Global epidemiology, ecology and control of soil-transmitted helminth infections," Advances in Parasitology, vol. 62, pp. 221-261, 2006.

[29] J. Bethony, J. Chen, S. Lin et al., "Emerging patterns of hookworm infection: influence of aging on the intensity of Necator infection in Hainan Province, People's Republic of China," Clinical Infectious Diseases, vol. 35, no. 11, pp. 1336-1344, 2002.

[30] S. Brooker, A. Jardim-Botelho, R. J. Quinnell et al., "Age-related changes in hookworm infection, anaemia and iron deficiency in an area of high Necator americanus hookworm transmission in south-eastern Brazil," Transactions of the Royal Society of Tropical Medicine and Hygiene, vol. 101, no. 2, pp. 146-154, 2007.

[31] E. C. Strunz, D. G. Addiss, M. E. Stocks, S. Ogden, J. Utzinger, and M. C. Freeman, "Water, sanitation, hygiene, and soiltransmitted helminth infection: a systematic review and metaanalysis," PLoS Medicine, vol. 11, no. 3, article e1001620, 2014. 

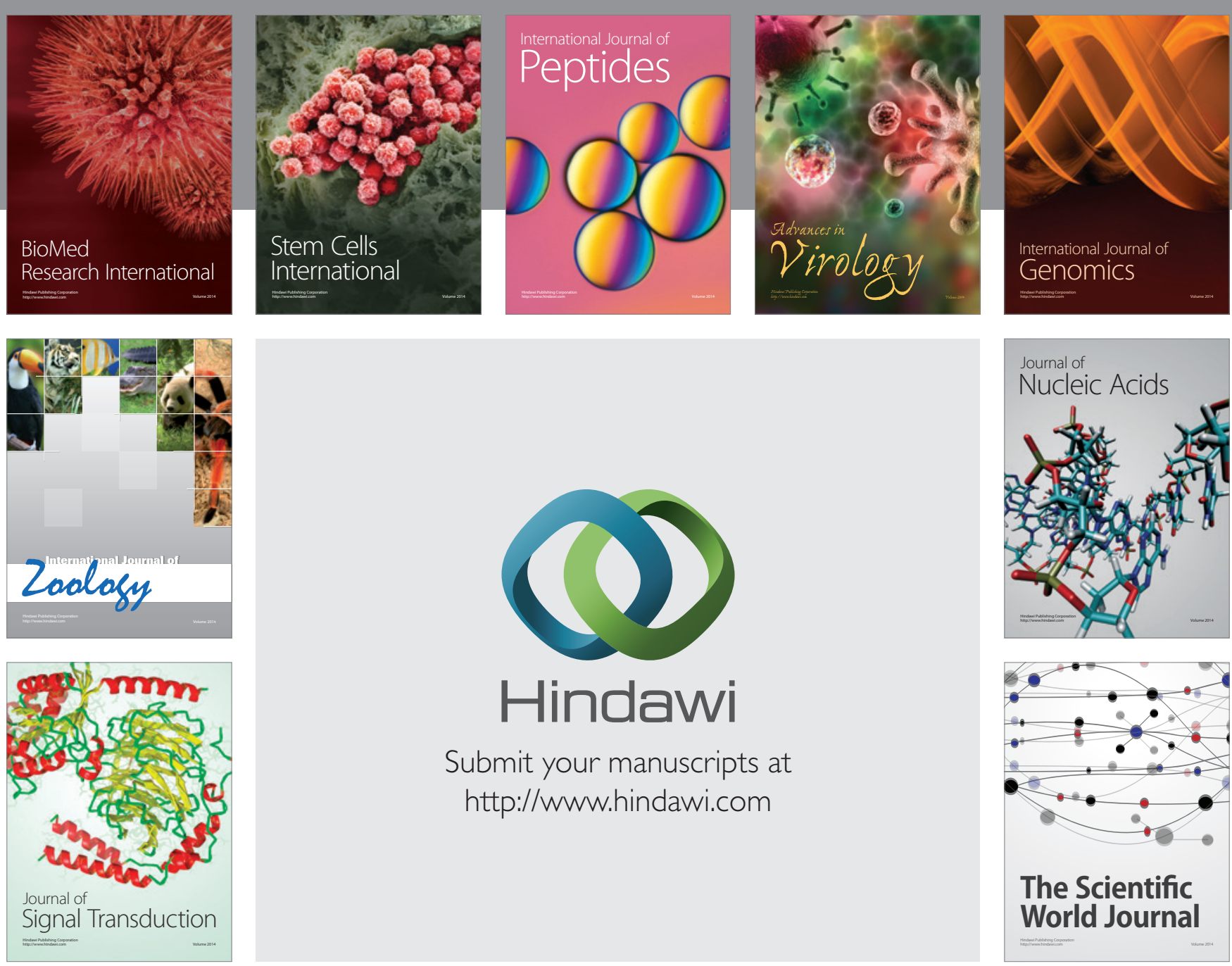

Submit your manuscripts at

http://www.hindawi.com
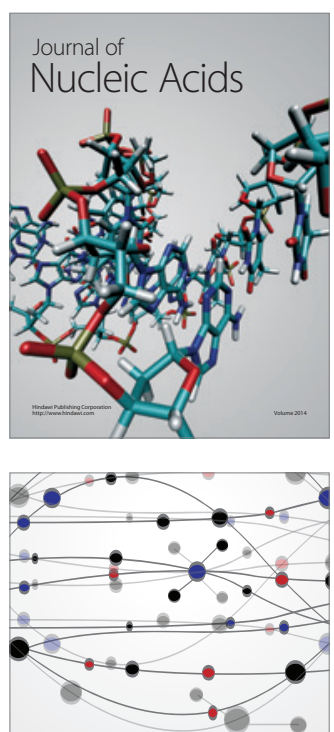

The Scientific World Journal
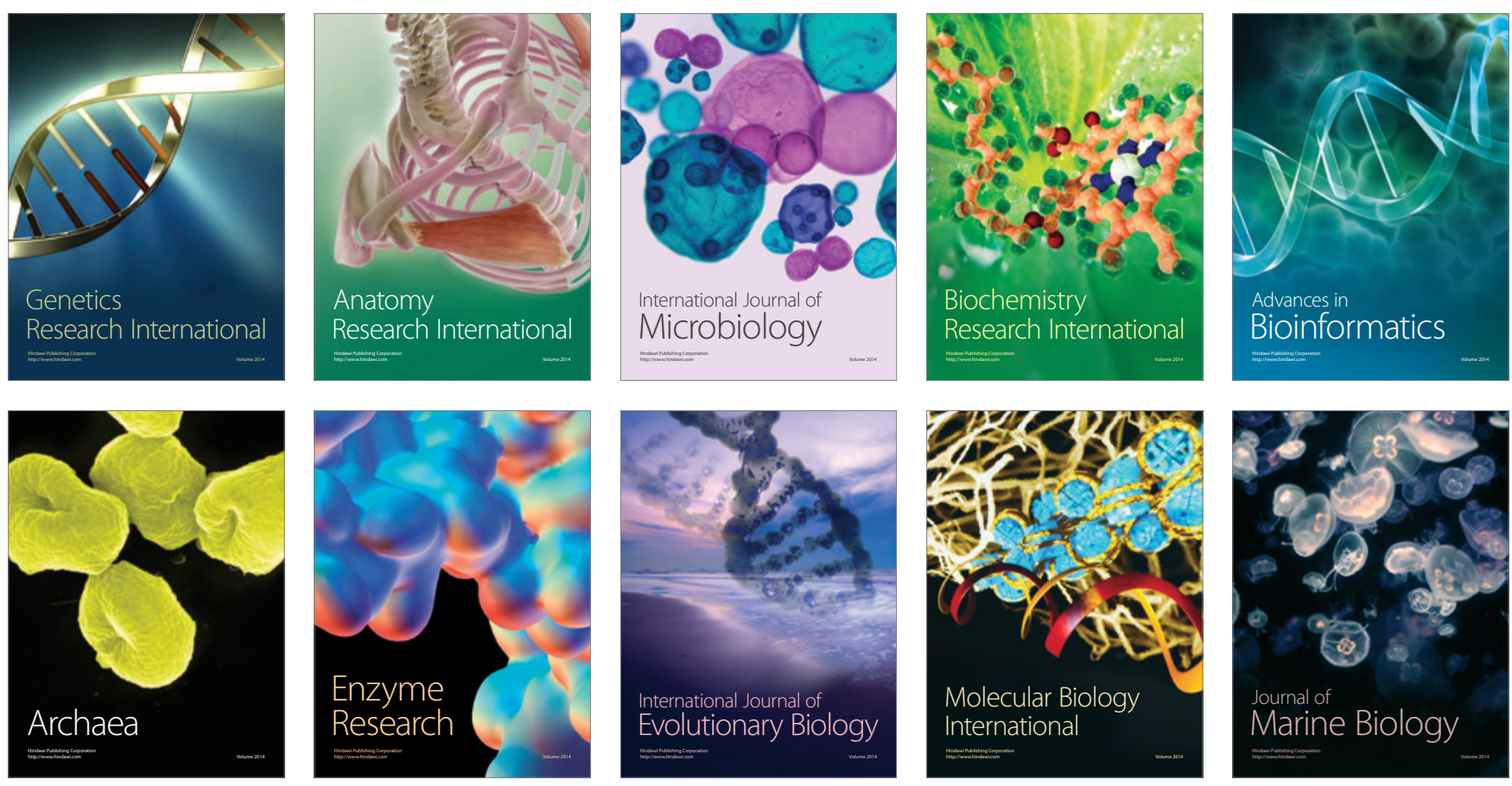